\title{
EXTRACCIÓN Y CARACTERIZACIÓN DEL ALGINATO DE SODIO DE LA MACROALGA Macrocystis pyrifera
}

\author{
Yerald Ore B., E. Renzo Pichilingue L., Ana C. Valderrama Negrón ${ }^{* a}$
}

\begin{abstract}
RESUMEN
En el presente trabajo se propuso un proceso de extracción de alginato de sodio, el cual fue obtenido a partir de la macroalga parda Macrocystis pyrifera recolectada en La PuntaCallao. El proceso de extracción presentó un rendimiento del $12 \%$ y el alginato obtenido fue caracterizado mediante las técnicas de IR-TF y RMN ${ }^{13} \mathrm{C}$ en estado sólido, el cual fue comparado con un alginato comercial. Se ha llevado a cabo la evaluación de la presencia de bloques manurónicos y gulurónicos IR-TF, mediante el uso de la 2da derivada obteniendo señales características a $894 \mathrm{~cm}^{-1}$ para $\mathrm{C}_{1}-\mathrm{H} \alpha-\mathrm{L}$-gulurónico del alginato extraído de la macroalga Macrocystis pyrifera $(\mathrm{AlgM})$ y a $885 \mathrm{~cm}^{-1}$ para la región anomérica $\mathrm{C}_{1}-\mathrm{H}$ del $\beta-\mathrm{D}-$ manurónico para el alginato comercial de Sigma Aldrich (AlgC). Asimismo, se asignaron señales características para el espectro del alga a $902 \mathrm{~cm}^{-1}$ y a $812 \mathrm{~cm}^{-1}$ correspondiente a vibraciones de los bloques $\alpha-\mathrm{L}$-gulurónico. Mediante ${ }^{13} \mathrm{C}$ RMN en estado sólido se obtuvo un pico característico a 102,4 ppm para AlgM asociado al carbono anomérico $\mathrm{C}_{1}-\mathrm{H} \alpha-\mathrm{L}-$ gulurónico, mientras que en $\mathrm{AlgC}$ se visualizaron dos picos a 101,8 ppm y a 99,8 ppm asignados a $\mathrm{C}_{1}-\mathrm{H} \alpha-\mathrm{L}$-gulurónico y $\mathrm{C}_{1}-\mathrm{H} \beta-\mathrm{D}$-manurónico, respectivamente, indicando así la presencia tanto de bloques $\mathrm{G}$ y bloques $\mathrm{M}$ en el alginato comercial.
\end{abstract}

Palabras clave: alginato, extracción, Macrocystis pyrifera, ${ }^{13} \mathrm{C}$ RMN en estado sólido.

\section{EXTRACTION AND CHARACTERIZATION OF SODIUM ALGINATE FROM THE MACROALGAE Macrocystis pyrifera}

\begin{abstract}
In the present work, it was proposal a process for the extraction of sodium alginate, which was obtained from the brown macroalgae Macrocystis pyrifera collected in La Punta-Callao. The extraction process presented a yield of $12 \%$ and the alginate obtained was characterized by FT-IR and ${ }^{13} \mathrm{C}$ NMR techniques in solid state, which was compared with a commercial alginate. The evaluation of the presence of mannuronic and guluronic blocks has been carried out through the use of the 2 nd derivative, obtaining characteristic signals at $894 \mathrm{~cm}^{-1}$ for $\mathrm{C}_{1}-\mathrm{H}$
\end{abstract}

\footnotetext{
a LIBIPMET, Facultad de Ciencias, Universidad Nacional de Ingeniería, Av. Túpac Amaru-Rímac, Lima, Perú, anitacvn29@yahoo.com.mx
} 
$\alpha-\mathrm{L}-$ guluronic in $\mathrm{AlgM}$ and at $885 \mathrm{~cm}-1$ for $\mathrm{C}_{1}-\mathrm{H}$ anomeric region of the $\beta$-D-mannuronic in AlgC. Moreover, characteristic signals were assigned for the algae spectrum at $902 \mathrm{~cm}^{-1}$ and at $812 \mathrm{~cm}^{-1}$ corresponding to vibrations of the $\alpha-\mathrm{L}-$ guluronic blocks. By ${ }^{13} \mathrm{C}$ NMR in solid state, a characteristic peak was obtained at $102.4 \mathrm{ppm}$ for $\mathrm{AlgM}$ associated with the anomeric carbon $\mathrm{C}_{1}-\mathrm{H} \alpha$ - L-guluronic, while in $\mathrm{AlgC}$ two peaks were visualized at $101.8 \mathrm{ppm}$ and 99.8 ppm assigned to the $\mathrm{C}_{1}-\mathrm{H} \alpha-\mathrm{L}-$ guluronic and $\mathrm{C}_{1}-\mathrm{H} \beta-\mathrm{D}-$ mannuronic respectively thus indicating the presence of both $\mathrm{G}$ blocks and $\mathrm{M}$ blocks in commercial alginate.

Key words: Alginate, Extraction, Macrocystis pyrifera, ${ }^{13} \mathrm{C}$ NMR in solid state.

\section{INTRODUCCIÓN}

Actualmente las algas marinas constituyen un sector importante de la economía mundial, esto se debe a las múltiples aplicaciones que tienen, entre ellas su uso en la industria alimentaria y biotecnológica. Estas, son fuente principal de ficocoloides, siendo los más representativos el agar-agar, fucanos, carragenano y alginato ${ }^{1}$.

Las algas pardas se caracterizan por encontrarse en mares templados o subpolares, predominando estas en la zona intermareal, caracterizadas por el tamaño de sus tallos y conformando así extensos bosques marinos, en donde la macroalga Macrocystis pyrifera es una de las especies más abundante del litoral peruano y a nivel mundia ${ }^{12}$.

Asimismo, esta especie es uno de los insumos de mayor demanda en países tales como EE.UU., Japón, China, Francia y Noruega para la producción de alginato de sodio ${ }^{3}$.

El alginato es un polisacárido el cual es obtenido a partir de las macroalgas pardas y está principalmente compuesto por los ácidos $\beta$-D-manurónico (M) y $\alpha$-L-gulurónico (G) figura 1 , los cuales se encuentran distribuidos de manera aleatoria en la estructura de la cadena polimérica. Además, el alginato puede presentar secuencias de monómeros de los residuos $\mathrm{M}$ y G, así como su alternancia de los mismos obteniendo de esta manera bloques $\mathrm{M}$ y bloques G (homopoliméricos), y bloques MG (heteropolimérico) ${ }^{4}$.

La relación del porcentaje de la distribución de los bloques M y G depende de la especie de la cual se obtiene el alginato. Asimismo, parámetros como la localización geográfica, hábitat, y especie determinan la distribución de estos grupos teniendo efecto en las propiedades fisicoquímicas de acuerdo a la relación $\mathrm{M} / \mathrm{G}$ del alginato obtenido ${ }^{5}$.

Por otro lado, las etapas para llevar a cabo la extracción del alginato influyen en el tipo de alginato obtenido, teniendo un efecto en el tamaño molecular, variación en el contenido de los ácidos urónicos, contenido de iones metálicos, y la presencia de polifenoles ${ }^{6}$. 


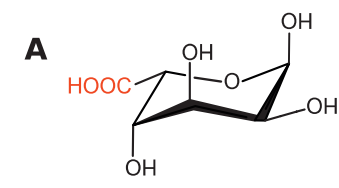

Ácido gulurónico

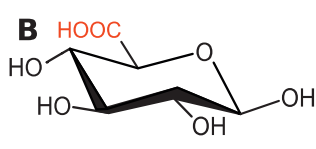

Ácido manurónico

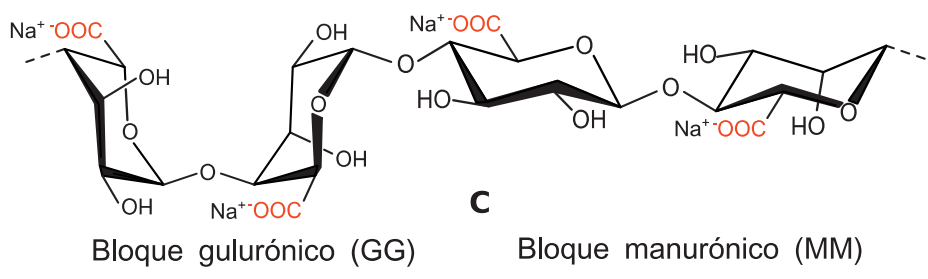

Figura 1. (A) Ácido gulurónico. (B) Ácido manurónico. (C) Composición del alginato con bloques GG y Bloques $\mathrm{MM}^{4}$.

En consecuencia, estas propiedades podrían definir el uso versátil de este polisacárido en distintos campos, así como sus derivados, presentando actividad biológica, biodegradabilidad y biocompatibilidad en sistemas vivos, en su actividad como agente anti-incrustante, intercambiador iónico (para un $\mathrm{M} / \mathrm{G}<1$ ), entre otros, fines de aplicación industrial ${ }^{4,7}$.

Es de este modo que, a lo largo de los últimos años, se han propuesto distintas metodologías para la extracción y purificación del alginato, mediante la obtención de ácido algínico ${ }^{8}$, a partir de la formación de geles mediante $\mathrm{CaCl}_{2}{ }^{9}$, implementación de un pre-tratamiento entre otros, las cuales en su mayoría se enfocan en optimizar el rendimiento de la extracción.

En el presente trabajo, se ha propuesto un proceso de extracción del alginato a partir del método alcalino y la formación de geles haciendo uso de $\mathrm{CaCl}_{2}$, teniendo como principal objetivo la caracterización del biopolímero mediante técnicas de IR-TF y su segunda derivada, y mediante 13C RMN en estado sólido para evaluar el contenido de los bloques $\mathrm{M}$ y $\mathrm{G}$ presentes en el alginato, lo cual nos permitirá estimar si la relación $\mathrm{M} / \mathrm{G}$ es menor o mayor a 1 .

\section{PARTE EXPERIMENTAL}

\section{MATERIALES}

El alginato de sodio (AlgC) fue adquirido de Sigma Aldrich Chemistry (St. Louis, MO, USA), Los solventes utilizados fueron de grado analítico adquiridos de MERCK (Darmstadt, Germany); La Macrocystis pyrifera fue colectada entre 5-10 m de profundidad a $16^{\circ} \mathrm{C}$ en la La Punta-Callao. Una vez llevado a cabo esto, se lavaron y almacenaron a $-20^{\circ} \mathrm{C}$. Las hojas que han sido usadas como materia prima para la producción de alginato fueron cortadas en trozos pequeños y secados en una estufa a $70^{\circ} \mathrm{C}$ por $3 \mathrm{~h}$. Finalmente, esta fue molida a un tamaño menor a $1 \mathrm{~mm}$. 


\section{EXTRACCIÓN DEL ALGINATO}

Se tomaron $50 \mathrm{~g}$ de alga pulverizada y se procedió a colocarla en una solución de n-hexano en relación (1:3) por 2 horas. Luego los trozos desengrasados (por el tratamiento con n-hexano) se colocaron en una mezcla etanol/formaldehido (4:1) dejando reposar por $72 \mathrm{~h}$. El producto obtenido fue tratado con carbonato de sodio al $3 \%$ a $90^{\circ} \mathrm{C}$ por $4 \mathrm{~h}$ (proceso de extracción alcalino $)^{10}$. Finalmente se dializó y liofilizó la fase líquida obtenida del proceso de extracción.

Asimismo, la purificación fue llevada a cabo mediante el uso de $\mathrm{CaCl}_{2}$ (formación de geles). A partir del producto obtenido se realizó un intercambio de la forma sal/ácida del alginato, variando el $\mathrm{pH}$ alcalino/ácido. Finalmente, el producto obtenido fue dializado y liofilizado, obteniendo un sólido blanco, $\mathrm{AlgM}^{6,8,11}$.

\section{ESPECTROSCOPIA INFRARROJA CON TRANSFORMADA DE FOURIER (IR-TF)}

El espectro infrarrojo fue obtenido usando un FT-IR IRPrestigy-21 mediante el método de reflactancia total atenuada (ATR). Los espectros fueron medidos (64 scans/muestra) en el rango de 4000-700 $\mathrm{cm}^{-1}$, con una resolución de 4,0 $\mathrm{cm}^{-1}$. La derivatización incluyó el algoritmo de Savitzky-Golay con 23 puntos de suavizado y todos los espectros fueron analizados con el software OriginLab 9.0.

\section{${ }^{13}$ C RMN EN ESTADO SÓLIDO}

El análisis de $\mathrm{RMN}{ }^{13} \mathrm{C}$ en estado sólido fue llevado a cabo usando un equipo Bruker Advance III-400, operando a 9,4 T de campo magnético, $v(1 \mathrm{H})=400 \mathrm{MHz}$. La ${ }^{13} \mathrm{C}$ frecuencia de resonancia fue de $100.57 \mathrm{MHz}$ con un pulso de secuencia (polarización cruzada con un ángulo mágico de spin, CP-MAS) y con una supresión total de banda (CP-MAS-TOSS). El alto poder de desacoplamiento del campo fue de $83.3 \mathrm{KHz}[\mathrm{P} 90(1 \mathrm{H})=3 \mu \mathrm{s}]$ y se usó “adamantano" como patrón de referencia externo para ajustar el desplazamiento del ${ }^{13} \mathrm{C}$.

\section{RESULTADOS Y DISCUSIÓN}

\section{EXTRACCIÓN DEL ALGINATO}

El proceso de extracción fue propuesto a partir de los trabajos de Matsuhiro et al. ${ }^{12}$. $\mathrm{y}$ Hernández-Carmona et al. ${ }^{10}$ el cual consistió en 3 etapas; i. Pre-tratamiento, ii. Extracción alcalina y iii. Purificación.

El primer paso fue someter el alga pulverizada a un pre-tratamiento, que consistió en un desengrasado a partir del uso de n-hexano, seguido de una eliminación de metabolitos (taninos, polifenoles, etc.) mediante un tratamiento con la mezcla etanol/formaldehido en relación de 4/1. A partir de la muestra obtenida, se procedió a llevar a cabo la extracción en medio alcalino (carbonato de sodio al $3 \%$ ) el cual permitió extraer los polisacáridos presentes en la pared celular del alga, manteniendo una temperatura de $90^{\circ} \mathrm{C}$ por 4 horas, obteniéndose finalmente así un extracto impuro (figura 2) del cual se aislaron 2 fases mediante centrifugación por 30 min y finalmente el líquido obtenido, fue liofilizado para su pronta purificación. 
De la purificación se obtuvo el ácido algínico, después de remover las impurezas presentes, ya que no solamente podríamos a ver aislado alginato de sodio el cual es el biopolímero en interés sino también, hemicelulosa, fucanos, carbohidratos, entre otros ${ }^{13}$. Es de este modo que se implementó dentro del proceso de purificación, la formación de geles con $\mathrm{Ca}+2$ (para la selectividad del alginato) y procesos de diálisis y centrifugación para la mayor purificación del alginato ${ }^{14}$. Obteniendo, de este modo, un material blanquecino, como podemos observar en la figura 3.

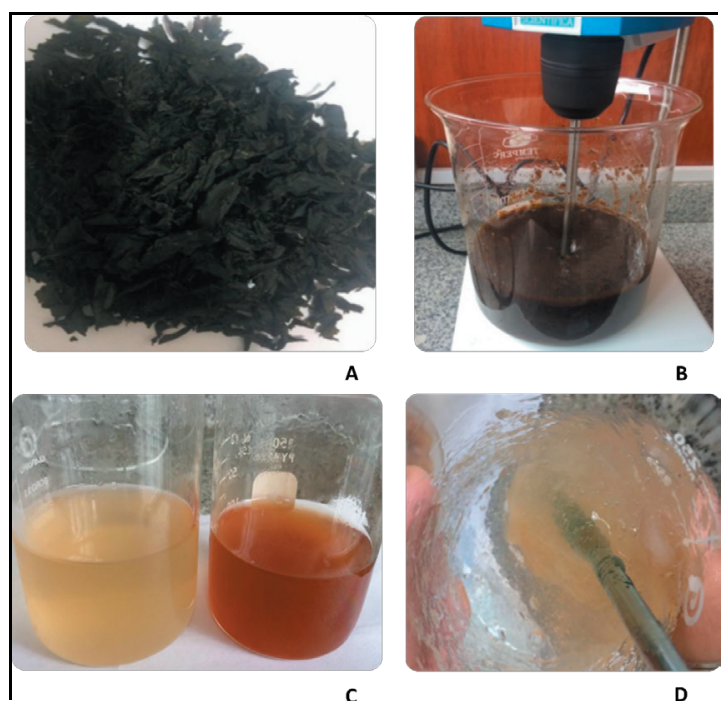

Figura 2. Extracción de alginato. (A) Materia prima, alga. (b) Tratamiento alcalino. (C) Comparación antes y después del alginato extraído y su purificación. (D) Intercambio de kps, en el proceso de purificación.

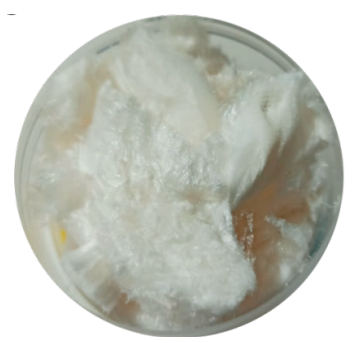

Figura 3. Alginato extraído de la macroalga Macrocystis pyrifera. 
El rendimiento obtenido del proceso de extracción propuesto del alga fue del $12 \%$ para la Macrocystis pyrifera. El protocolo propuesto permitió obtener un alginato de coloración blanquecina, lo que indicaría que en el proceso se pudo eliminar gran contenido de polifenoles, presentes en el alga parda de partida. A pesar de esto, el rendimiento no fue tan alto a comparación de las obtenidas para otras algas ya reportadas ${ }^{15,16}$. Esto, posiblemente es debido, al ambiente natural, estación de la cosecha ${ }^{3}$, entre otros factores que involucran al entorno del crecimiento del alga. Asimismo, otro factor que influiría en el rendimiento, serían los pasos tomados en cada etapa del proceso de extracción, ya que cada tratamiento es determinante y no solo involucra la extracción del alginato, sino también la influencia en la degradación del mismo, debido al tratamiento de hidrólisis (ácida o básica) ${ }^{17}$ que ocurre a lo largo de la cadena del biopolímero, disminuyendo así su masa molecular modificando las propiedades que podría presentar, influenciadas estas por la secuencia de los bloques $\mathrm{M}$ y $\mathrm{G}$ y su proporción $\mathrm{M} / \mathrm{G}$ presente en la cadena polimérica.

\section{ESPECTROSCOPIA INFRARROJA CON TRANSFORMADA DE FOURIER (IR-TF)}

En la figura 4 presentamos los espectros del alga de partida (Macrocystis pyrifera), el alginato extraído $(\mathrm{A} \lg \mathrm{M})$ y el alginato comercial de Sigma Aldrich $(\mathrm{AlgC})$, de este modo podemos asignar los grupos funcionales acorde a las vibraciones que presenta la figura 4. En este caso las señales características del alginato más representativas son:

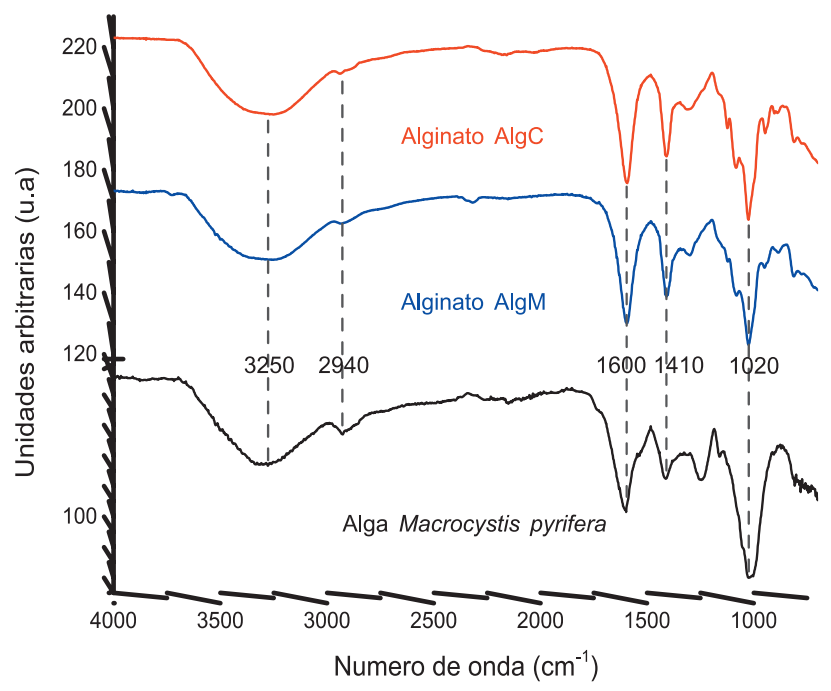

Figura 2. Espectro IR-TF del Alga Macrocystis pyrifera, Alginato AlgM y Alginato AlgC. 
La señal alrededor de $3250 \mathrm{~cm}^{-1}$ y $2940 \mathrm{~cm}^{-1}$ correspondientes a la vibración de estiramiento del grupo funcional $\mathrm{O}-\mathrm{H}$ y $\mathrm{C}-\mathrm{H}$, respectivamente, ambos presentes a lo largo de la cadena del alginato. Además, alrededor de $1600 \mathrm{~cm}^{-1}$ y $1410 \mathrm{~cm}^{-1}$ se observa la vibración antisimétrica y simétrica respectivamente, del grupo carboxilato. Entre 1300 a $1000 \mathrm{~cm}-1$ podemos asociar las señales características de las vibraciones de estiramiento de C-C-H, O-C-H, C-O-C, entre otros, asociados al entorno de la piranosa, siendo la más representativa la banda $1020 \mathrm{~cm}^{-1}$ asociada a la vibración de estiramiento C-O-C de la piranosa ${ }^{7}$.

Por otro lado, en el caso del espectro de la macroalga Macrocystis pyrifera, presentamos a $1540 \mathrm{~cm}^{-1}$ una banda atribuida a la vibración de estiramiento de grupo amida $(\mathrm{N}-\mathrm{H})$, esto debido a la presencia de proteínas. Asimismo, a $1750 \mathrm{~cm}^{-1}$ asignamos la señal del grupo $\mathrm{C}=\mathrm{O}$ el cual caracteriza a la presencia de lípidos. Finalmente, a $1250 \mathrm{~cm}^{-1}$ encontramos una banda que representa a la vibración de estiramiento del grupo $\mathrm{S}=\mathrm{O}$, característico a la presencia de otros polisacáridos presentes en el alga ${ }^{18}$.
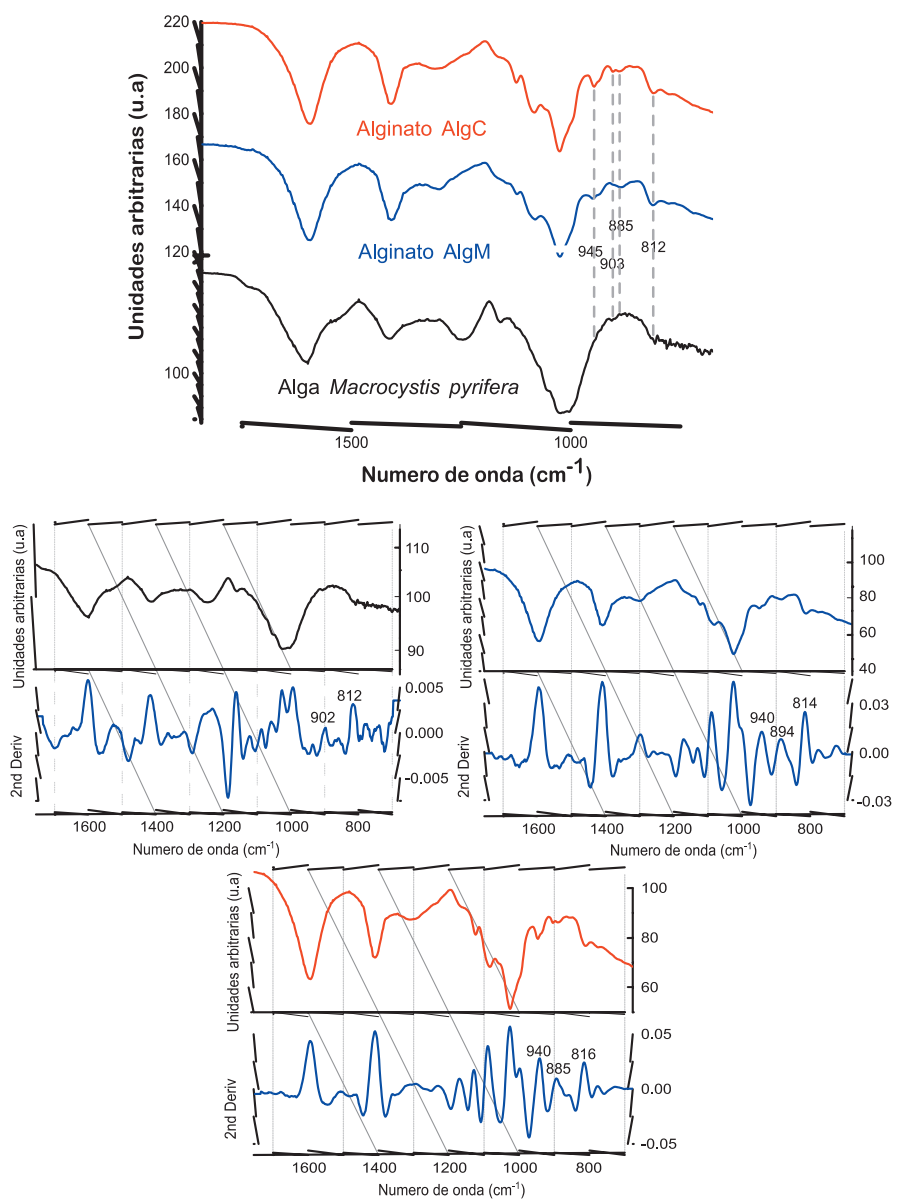

Figura 5. Espectro FT-IR y su segunda derivada en la región de la huella dactilar. 
En el entorno de la huella dactilar podríamos atribuir bandas asociadas a los bloques homopoliméricos tanto de GG y MM. Evaluando las señales entre 1200 a $750 \mathrm{~cm}^{-1}$ (figura 5) observamos bandas características a $945 \mathrm{~cm}^{-1}$, la cual es asignada a las vibraciones $\mathrm{C}-\mathrm{O}$, con contribución de C-C-O debido a la deformación vibracional de los residuos de los ácidos urónicos presentes en ambos alginatos. Las bandas a 903 y $885 \mathrm{~cm}^{-1}$ son asignadas a las vibraciones de deformación de $\mathrm{C}_{1}-\mathrm{H} \alpha-\mathrm{L}$-gulurónico y $\mathrm{C}_{1}-\mathrm{H}$ del $\beta-\mathrm{D}$-manurónico. Asimismo, a $812 \mathrm{~cm}^{-1}$ ambos alginatos presentan esta banda característica de deformación de la piranosa de los bloques $\alpha-\mathrm{L}$-gulurónico ${ }^{7,14}$.

Por otro lado, para evaluar significativamente las diferencias que existen en estos espectros se procedió a realizar la 2da derivada (figura 5) para visualizar aquellas señales que no son fácilmente observables en el espectro común, tales como las interacciones para cada bloque MM y GG. Es así que tenemos para AlgM la banda a $894 \mathrm{~cm}^{-1}$ la cual es característica a la vibración de la región anomérica $\mathrm{C}_{1}-\mathrm{H} \alpha-\mathrm{L}$-gulurónico a diferencia de $\mathrm{AlgC}$ el cual presenta una banda a $885 \mathrm{~cm}^{-1}$ característico a la vibración de la región anomérica $\mathrm{C}_{1}-\mathrm{H}$ del $\beta-\mathrm{D}-$ manurónico ${ }^{7,11}$.

Ambos alginatos presentaron señales a $940 \mathrm{~cm}^{-1}$, lo cual fue asignado a la vibración de estiramiento C-O. Finalmente, en el caso del espectro de la macroalga, podemos observar las señales a $902 \mathrm{~cm}^{-1}$ y a $812 \mathrm{~cm}^{-1}$ característicos a la deformación de la piranosa de los bloques $\alpha-\mathrm{L}-$ gulurónico pudiendo interpretar la presencia de los bloques GG antes de llevar a cabo el proceso de extracción del alginato.

Por lo tanto, de los resultados obtenidos, tenemos que el AlgM y AlgC presentan diferencias respecto a la relación del contenido de bloques $\mathrm{M}$ y $\mathrm{G}$ ya que se han observado diferencias en las bandas que corresponden $\mathrm{C}_{1}-\mathrm{H} \alpha-\mathrm{L}-$ gulurónico y $\mathrm{C}_{1}-\mathrm{H}$ del $\beta-\mathrm{D}$-manurónico presentes en la cadena polimérica, los cuales serán mejor diferenciados mediante el ensayo de Resonancia Magnética Nuclear.

\section{RESONANCIA MAGNÉTICA NUCLEAR EN ESTADO SÓLIDO}

La figura 6 muestra el espectro de RMN 13C en estado sólido del AlgM y AlgC 


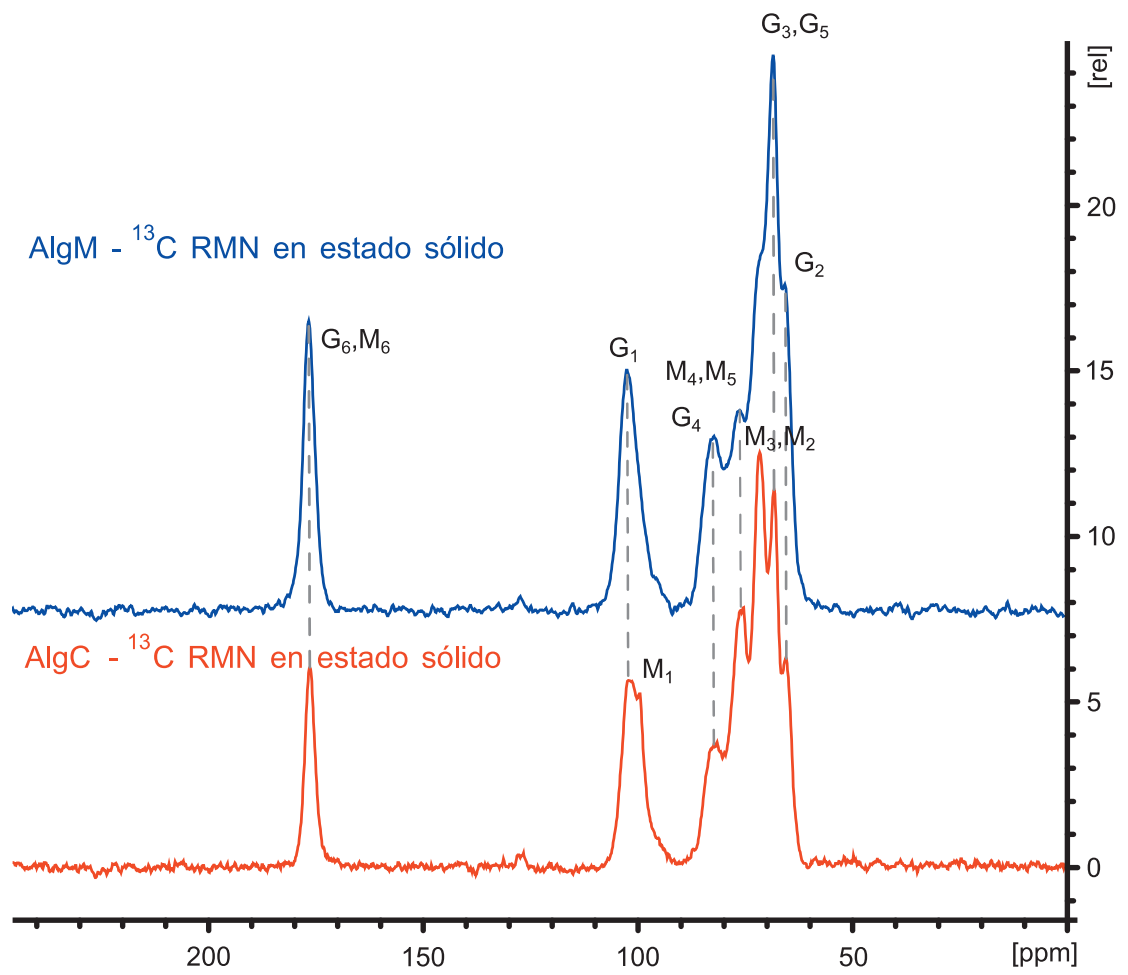

Figura 6. Análisis $\mathrm{RMN}{ }^{13} \mathrm{C}$ en estado sólido de $\mathrm{AlgM}$ y AlgC.

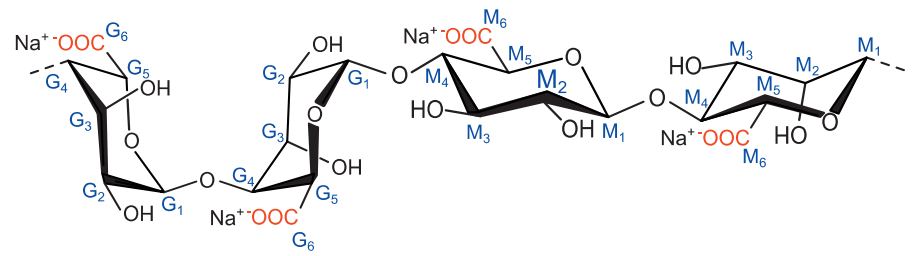

Figura 7. Estructura del alginato con cada carbono enumerado.

En la tabla 1 se muestran los desplazamientos químicos obtenido a partir del $\mathrm{RMN}{ }^{13} \mathrm{C}$ en estado sólido de la figura 6.

- Los grupos carboxilatos (160 - $180 \mathrm{ppm})$

- $\quad$ Puente glicosídico $(90-110$ ppm)

- $\quad$ Entorno de la piranosa $(50-80 \mathrm{ppm})$ 
Tabla 1. Asignación de los espectros RMN 13C en estado sólido de la figura 6 tomando en consideración la estructura del alginato mostrado en la figura 7.

\begin{tabular}{|c|c|c|c|c|c|c|c|c|}
\hline Ambiente Químico & Carbonilo & Ano & érico & \multicolumn{5}{|c|}{ Piranosa } \\
\hline $\begin{array}{c}\text { Carbones de los grupos } \\
\text { manurónicos (M) y } \\
\text { gulurónicos (G) }\end{array}$ & $\mathrm{G}_{6} \quad \mathrm{M}_{6}$ & $\mathrm{G}_{1}$ & $\mathrm{M}_{1}$ & $\mathrm{G}_{4}$ & $\mathrm{M}_{4} \quad \mathrm{M}_{5}$ & $\begin{array}{ll}M_{3} & M_{2}\end{array}$ & $\mathrm{G}_{3}$ & $\mathrm{G}_{2}$ \\
\hline D. Químico AlgC (ppm) & 176,4 & 101,8 & 99,4 & 82,1 & 75,7 & 71,7 & 68,3 & 65,5 \\
\hline D. Químico AlgM (ppm) & 176,1 & 102,4 & --- & 81,6 & 75,6 & 71,6 & 68,2 & 65,4 \\
\hline $\begin{array}{c}\text { Desplazamiento Químico } \\
(\mathrm{ppm})^{19}\end{array}$ & 176,3 & 102,2 & 99,5 & 82,8 & 76,4 & 71,6 & 68,4 & 65,5 \\
\hline
\end{tabular}

Evaluando las diferencias entre AlgM y AlgC, obtenido de la tabla 1 y de la figura 7, tenemos que la presencia de los carbones $\mathrm{G}$ son más representativos en $\mathrm{AlgM}$ que en $\mathrm{AlgC}$. Como se ha mencionado anteriormente, el entorno entre 110 - 90 ppm evalúa los carbones presentes en el puente glicosídico el cual determina la interacción y la formación de los bloques presentes. A 102,4 ppm tenemos al carbono anomérico $\mathrm{C}_{1}-\mathrm{H} \alpha-\mathrm{L}$-gulurónico que se observa en el AlgM desplazándose esta señal a un campo más bajo que el que presenta $\mathrm{AlgC}$ a 101,8 ppm. En el espectro de este último también se observa la señal del carbono anomérico C1-H del $\beta$-D-manurónico a 99,8 ppm indicando así la presencia característica de los bloques $\mathrm{M}$, lo que no se observa en el AlgM.

Asimismo, evaluando el entorno de la piranosa presentamos señales de los carbones $\mathrm{G}_{3}-\mathrm{G}_{5}$ los cuales son más intensos que las señales de $\mathrm{M}_{3}$ y $\mathrm{M}_{2}$ para el AlgM, soportando así la propuesta que este alginato presenta un mayor \% de grupos G. A diferencia de esto tenemos que en $\mathrm{AlgC}$, se presenta señales más intensas alrededor de $\mathrm{M}_{3} \mathrm{y}_{2} \mathrm{y}$ el pico que caracteriza a $\mathrm{G}_{3}-\mathrm{G}_{5}$ la señal es casi indiferenciable.

Por ende, a partir de estos análisis podemos interpretar que la relación M/G para el AlgM es $<1$ y para el $\mathrm{Alg} C>1$. De la misma manera, nos permite proponer que para el AlgM existe un mayor porcentaje de bloques $\mathrm{G}$ a diferencia del AlgC. A partir de estos resultados, con el AlgM se podría obtener materiales que se beneficien a partir de la propiedad de reticulación iónica a diferencia de $\mathrm{AlgC}$, debido a la mayor presencia de bloques GG en la estructura del alginato de AlgM. A pesar de ello, la presencia de bloques $\mathrm{M}$ a diferencia de los bloques $\mathrm{G}$, son menos reactivos y por tanto son más estables frente a procesos de modificación y degradación ${ }^{7,20}$. 


\section{CONCLUSIONES}

Se logró extraer alginato sodio, el cual presentó un rendimiento del $12 \%$. Para el alginato obtenido de la macroalga (AlgM) se logró demostrar la presencia de carbones y grupos que asocian a la región anomérica del $\mathrm{C}_{1}-\mathrm{H} \alpha-\mathrm{L}$-gulurónico. Asimismo, el espectro del alga, mostró bandas a $902 \mathrm{~cm}^{-1}$ y a $812 \mathrm{~cm}^{-1}$ característicos de los bloques $\mathrm{G}$ lo que indicaría que esta macroalga es rica en grupos gulurónicos a diferencia del alginato comercial (AlgC). Finalmente, mediante los resultados obtenidos se interpretaría que la relación $\mathrm{M} / \mathrm{G}$ para $\mathrm{A} \lg \mathrm{M}$ seria $<1$ y para $\mathrm{Alg} \mathrm{C}>1$.

Es así que ambas técnicas (IR-TF y RMN en estado sólido) nos permiten diferenciar la presencia de bloques $\mathrm{M}$ y $\mathrm{G}$, así como poder interpretar antes de llevar a cabo la extracción de alginato, la presencia de bloques gulurónicos en la macroalga.

\section{AGRADECIMIENTO}

Al Lic. Ronny G. Huamaní Palomino, por su contribución en las discusiones del IR-TF y ${ }^{13} \mathrm{C} \mathrm{RMN}$ en estado sólido. Asimismo, al Vicerrectorado de Investigación y a la Oficina de Gestión de la Investigación de la Universidad Nacional de Ingeniería por el financiamiento del proyecto FC-F-13-2018.

\section{REFERENCIAS BIBLIOGRÁFICAS}

1. Øverland M, Mydland LT, Skrede A. Marine macroalgae as sources of protein and bioactive compounds in feed for monogastric animals. J Sci Food Agric. 2019; 99(1):1324.

2. Baweja P, Kumar S, Sahoo D, Levine I. Biology of Seaweeds. In: Fleurence J, Levine I. Seaweed in Health and Disease Prevention. London: Academic Press; 2016. p 41-106.

3. Peteiro C. Alginate Production from Marine Macroalgae, with Emphasis on Kelp Farming. In: Rehm B, Moradali M. (eds) Alginates and Their Biomedical Applications. Singapore: Springer; 2018. p 27-66.

4. Lee KY, Mooney DJ. Alginate: Properties and biomedical applications. Prog Polym Sci. 2012;37(1):106-126.

5. Arvizu-Higuera DL. Parameters afecting the conversion of alginic acid to sodium alginate. Ciencias Mar. 2002; 28(1):27-36.

6. Hernández-Carmona G, Freile-Pelegrín Y, Hernández-Garibay E. Conventional and alternative technologies for the extraction of algal polysaccharides. In: Dominguez $\mathrm{H}$. Functional Ingredients from Algae for Foods and Nutraceuticals. Cambridge, United Kingdom: Woodhead Publishing; 2013. p 475-516.

7. Huamaní-Palomino RG, Jacinto CR, Alarcón H, Mejía IM, López RC, Silva D de O, et al. Chemical modification of alginate with cysteine and its application for the removal of $\mathrm{Pb}$ (II) from aqueous solutions. Int J Biol Macromol. 2019;129: 1056-1068. 
8. Chandía NP, Matsuhiro B, Mejías E, Moenne A. Alginic acids in Lessonia vadosa: Partial hydrolysis and elicitor properties of the polymannuronic acid fraction. J Appl Phycol. 2004;16(2):127-133.

9. Reyes Tisnado R, Hernández Carmona G, Rodríguez Montesinos E, Arvizu Higuera D, López Gutiérrez F. Food grade alginates extracted from the giant kelp Macrocystis pyrifera at pilot-plant scale. Rev Investig Mar. 2005;26(3):185-192.

10. Hernández-Carmona G, McHugh DJ, Arvizu-Higuera DL, Rodríguez-Montesinos YE. Pilot plant scale extraction of alginates from Macrocystis pyrifera 3. Precipitation, bleaching and conversion of calcium alginate to alginic acid. J Appl Phycol. 2002; 14(6):445-451

11. Chandia NP, Matsuhiro B, Vásquez AE. Alginic acids in Lessonia trabeculata: Characterization by formic acid hydrolysis and FT-IR spectroscopy. Carbohydr Polym. 2001;46(1):81-87.

12. Martínez-Gómez F, Guerrero J, Matsuhiro B, Pavez J. In vitro release of metformin hydrochloride from sodium alginate/polyvinyl alcohol hydrogels. Carbohydr Polym. 2017; 155:182-191.

13. García-Vaquero M, Rajauria G, O’Doherty J V., Sweeney T. Polysaccharides from macroalgae: Recent advances, innovative technologies and challenges in extraction and purification. Food Res Int. 2017; 99(Part 3): 1011-1020.

14. Leal D, Matsuhiro B, Rossi M, Caruso F. FT-IR spectra of alginic acid block fractions in three species of brown seaweeds. Carbohydr Res. 2008;343(2):308-316.

15. Hagen Rødde RS, Vårum KM, Larsen BA, Myklestad SM. Seasonal and geographical variation in the chemical composition of the red alga Palmaria palmata (L.) Kuntze. Bot Mar. 2004;47(2):125-133.

16. Rhein-Knudsen N, Ale MT, Ajalloueian F, Meyer AS. Characterization of alginates from Ghanaian brown seaweeds: Sargassum spp. and Padina spp. Food Hydrocoll. 2017; 71: 236-244.

17. Draget KI, Taylor C. Chemical, physical and biological properties of alginates and their biomedical implications. Food Hydrocoll. 2011;25(2):251-256.

18. Kleinübing SJ, Gaia F, Bertagnolli C, Da Silva MGC. Extraction of alginate biopolymer present in marine alga sargassum filipendula and bioadsorption of metallic ions. Mater Res. 2013; 16(2):481-488.

19. Salomonsen T, Jensen HM, Larsen FH, Steuernagel S, Engelsen SB. Direct quantification of $\mathrm{M} / \mathrm{G}$ ratio from 13C CP-MAS NMR spectra of alginate powders by multivariate curve resolution. Carbohydr Res. 2009;344(15):2014-2022.

20. Gómez CG, Rinaudo M, Villar MA. Oxidation of sodium alginate and characterization of the oxidized derivatives. Carbohydr Polym. 2007; 67(3): 296-304. 